\title{
Endocan, VEGF, FGF2 and PDGF Expression in Pituitary Tumors. A Precise Angiogenic Marker Relationship in the Context of Tumor Invasiveness
}

\section{Pengyu Tao}

Otorhinolaryngology Head and Neck Surgery/Neurosurgery Department, Xuanwu Hospital, Capital

Medical University, Beijing, China

\section{Sofia Perrone}

Centro de Investigaciones Basicas y Aplicadas. UNNOBA

\section{Gianina Demarchi}

Centro de Investigaciones Basicas y Aplicadas. UNNOBA

\section{Agustina Chimento}

Centro de Investigaciones Basicas y Aplicadas, UNNOBA

\section{Andres Cervio}

Instituto FLENI. Departamento de Neurocirugia, Departamento de Neuropatologia

\section{Gustavo E Sevlever}

FLENI: Fundacion para la Lucha contra las Enfermedades Neurologicas de la Infancia

\section{Silvia Ines Berner}

Clinica Santa Isabel, Servicio de Neurocirugia.

\section{Damasia Becu-Villalobos}

Instituto de Biología y Medicina Experimental: Instituto de Biologia y Medicina Experimental

\section{Armando Basso}

Fundacion de Neurociencias Aplicadas. Universidad de Buenos Aires

\section{Chen Ge}

Xuanwu Hospital. Beijing

\section{Feng Ling}

Xuanwu Hospital, Beijing

Qiuhang Zhang (D 13701267977@163.com )

Xuanwu Hospital Department of Neurosurgery

Carolina Cristina ( $\nabla$ carolina.cristina@nexo.unnoba.edu.ar)

Centro de Investigaciones y Transferencia del Noroeste de la Provincia de Buenos Aires https://orcid.org/0000-0003-3161-7305 
Keywords: Angiogenesis, endocan, invasion, pituitary, PDGF, FGF

Posted Date: January 20th, 2021

DOI: https://doi.org/10.21203/rs.3.rs-150356/v1

License: (c) (1) This work is licensed under a Creative Commons Attribution 4.0 International License. Read Full License 


\section{Abstract}

Background: Pituitary adenomas can become invasive and aggressive, and in turn produce tumor recurrence. Endocan or endothelial cell specific molecule-1 (ESM1) has been associated with angiogenesis and tumor growth in gliomas, and lung, kidney, liver among other cancers, but there is a scarcity of information regarding its role in pituitary adenomas. In the search for biomarkers of tumor behavior our objective was to determine the relationship between endocan (ESM1) mRNA expression and cavernous sinus invasion, and its association with gene expression of VEGF, FGF2 and PDGF angiogenic factors in human pituitary adenomas.

Methods: ESM1, VEGF, FGF2 and PDGF expression was determined by qRT-PCR in 28 pituitary tumor samples and tumor invasion was determined by the Knosp grade classification. Results were associated with clinical data.

Results: We found that ESM1 was expressed in 75\% of the tumors. FGF2 expression was higher in ESM1positive compared to ESM1-negative tumors, and a significant negative correlation in the expression of FGF2 and PDGF was found. Although ESM1 expression did not show any differences in invasive and non-invasive tumors, a positive correlation between ESM1 and Knosp grade was reached when grade 0-3 tumors were considered, suggesting its participation in the initial periods of tumor invasion. Finally, no difference in ESM1 expression was found between functioning and non-functioning adenomas, or patients age.

Conclusions: Our study points to a precise pattern of angiogenic factor expression in pituitary tumors, and its relation to invasive behavior, which should be considered in the tailoring of drug treatments for aggressive and resistant pituitary adenomas.

\section{Introduction}

Even if pituitary adenomas can become aggressive, recur after surgery and be locally invasive, they are not considered malignant tumors because less than $0.2 \%$ of them produce metastasis $(1,2)$.

Nevertheless, cavernous sinus invasion limits neurosurgical procedures usually making complete tumor resection impossible (3). In turn, parasellar extension, determined by pre-operative magnetic resonance image (MRI) analysis, is a cause of high recurrence after surgery.

Therefore, the search for aggressive behavior biomarkers remains a matter of key importance in the pituitary tumor field. In this context, angiogenic factors are candidate markers of invasion and aggressiveness. In our laboratory and others a marked correlation between angiogenic factors, their receptors and pituitary tumor invasiveness has been demonstrated $(4,5)$.

Vascular endothelial growth factor, VEGF, is the most potent cytokine that promotes angiogenesis and metastasis in several tumors (6-8). However, in the pituitary, as in other tumors, a concerted balance of angiogenic and antiangiogenic mediators regulates tumor behavior and needs to be deciphered (9). 
Endocan, the endothelial cell specific molecule-1 (ESM-1), was demonstrated to be associated with angiogenesis and tumor growth in lung, kidney and liver among other cancers (10-12). Moreover, endocan expression was correlated with high grade gliomas $(13,14)$. It has been proposed as a neovascularization marker, being stimulated by two other important angiogenic factors VEGF and fibroblast growth factor (FGF2) $(10,13)$, and, importantly, it is involved in the switch from dormant to fast growing and angiogenic tumors (15).

In pituitary tumors the role of endocan and its relation to other angiogenic markers has not been extensively approached. Two immunohistochemical studies associated endothelial cell expression of endocan with aggressive pituitary adenoma behavior $(16,17)$. But some controversies arose between both reports in relation to clinical data and endocan expression (18). Moreover, no associations between endocan and angiogenic markers beyond VEGF, were evaluated in human pituitary tumor specimens.

In this context, we investigated intratumoral ESM1 mRNA expression in 28 human pituitary tumor samples obtained after surgery. Endocan expression was analyzed in relation to clinical parameters (pituitary hormone production, sex, age and image data) and also to the expression of the angiogenic markers VEGF, FGF2 and platelet-derived growth factor (PDGF). PDGF is a growth factor that regulates cell growth and division, and plays a significant role in blood vessel formation, and the growth of blood vessels from already-existing ones in different cell types, with little information in pituitary adenoma development $(19,20)$. We also wished to unravel the relationship between endocan and cavernous sinus invasion which, as mentioned, limits the efficacy of neurosurgery. Sinus invasion was determined by Knosp grade classification, and only Knosp grade 3 and 4 determine invasive pituitary tumors (21). Furthermore, we analysed the correlation of endocan and angiogenic related factors in order to unravel an angiogenic signature related to tumor invasion in pituitary tumors.

This work provides new data in the context of recent therapies which envisage anti-angiogenesis treatment in aggressive pituitary tumors $(22,23)$.

\section{Materials And Methods}

\section{Patients}

Tissue samples from 28 patients diagnosed with pituitary adenomas were obtained from neurosurgeries conducted at Xuanwu Hospital, Beijing, China and from the neurosurgery division of FLENI Institute, Buenos Aires, Argentina. Patients were, at the time of the surgery between 27 and 78 years old (mean \pm $\mathrm{SD}=51.3 \pm 13.0$ years), and 12 were women and 17 were men Adenoma samples were previously classified according to clinical criteria and WHO classification into functioning tumors: somatotropinomas, corticotropinomas, and mixed adenomas secreting prolactinomas and growth hormone, or in non-functioning adenomas when no hormone secretion was detected (24). Twenty two non-functioning, 3 mixed, 2 somatotropinomas and 1 corticotropinomas were anaylsed. 
MRI coronal scanning was used to assess cavernous sinus invasion of the 28 patients who underwent surgery. The extension of invasion was determined with Knosp grade classification by the intercarotid line system as described $(2,21)$. Five grades are used to represent the extent of tumor invasion from grade 0 to 4 . According to the Knosp MRI grading system, grades 3-4 were considered to be invasive whereas grades $0-2$ were considered non-invasive. In the analysed samples Knosp grades ranged from 0 to 4; and 4 tumors were recurrent.

For subsequent mRNA expression analysis after surgery, samples were kept in RNAlater solution (Life Technologies).

\section{Ethics approval and consent to participate}

The project and informed consent document were approved by the respective Institutional Ethical Committees from Xuanwu Hospital, Beijing, China (\#2019-03), and from the neurosurgery division of FLENI Institute, Buenos Aires, Argentina (\#04-2918) and every patient was informed about the procedures of the project and approved and signed the respective informed consent for participation in this study. Patient privacy was always preserved.

\section{RNA extraction and cDNA synthesis.}

Total RNA from pituitary tumors samples was extracted as described previously $(25,26)$ by TRI reagent (Molecular Research Center, Inc) method. The RNA concentration was determined on the basis of absorbance at $260 \mathrm{nM}$, only samples with a ratio of absorbance of 260/280 nm $\sim 2.0$ was considered appropriate, and RNA integrity was evaluated by agarose gel electrophoresis. One $\mu \mathrm{g}$ of RNA was reversed transcribed in $20 \mu \mathrm{L}$ volume in the presence of $3 \mathrm{mM} \mathrm{MgCl}_{2}, 50 \mathrm{mM}$ Tris. $\mathrm{HCl}(\mathrm{pH}$ 8.3), $75 \mathrm{mM}$ $\mathrm{KCl}, 1 \mathrm{mM}$ deoxy-NTPs, $0.01 \mathrm{mM}$ DTT, 1 pM oligo(dT) ${ }_{15}$ primer (Biodynamics, Buenos Aires, Argentina), and $10 \mathrm{U}$ of MMLV reverse transcriptase (Invitrogen, CA, USA). Negative controls were prepared by omitting the reverse transcriptase or mRNA.

\section{Real time PCR}

Quantitative PCR was performed as previously described in (27). Sense and antisense oligonucleotide primers were designed with PrimerBlast (http://www.ncbi.nlm.nih.gov/tools/primer-blast/) software. Primer sequences were: ESM1 Sense 5' ACCTTCGGGATGGATTGCAG 3' and Antisense 5' GATGCCATGTCATGCTCCGT 3'; VEGF Sense 5' CTCCTCCACCATGCCAAGT 3' and Antisense 5' GCAGTAGCTGCGCGCTGATAGA 3'; FGF2 Sense 5' CCTGGCTATGAAGGAAGATGG 3' and Antisense 5' TCGTTTCAGTGCCACATACC 3'; PDGF Sense 5' TGCCTCTCCGCACTCACT 3' and Antisense 5' AGAACATGGGCGAGGTATCC 3'; and GAPDH Sense 5' TGATGACATCAAGAAGGTGGTGAA 3' and Antisense 5' TCCTTGGAGGCCATGTAGGCCAT 3'. Oligonucleotides were purchased from Biodynamics SRL.

Quantitative PCR was prepared using 3uL 5X HOT FIREPOL EvaGreen qPCR Mix plus (Solis Biodyne) $75 \mathrm{ng}$ cDNA and $0.5 \mu \mathrm{M}$ primers in a final volume of $15 \mu \mathrm{L}$. After denaturation at $95 \mathrm{C}$ during $15 \mathrm{~min}$, the 
cDNA products were amplified with 40 cycles, $20 \mathrm{~s}$ at $95^{\circ} \mathrm{C}, 60 \mathrm{~s}$ at $55,5-62^{\circ} \mathrm{C}$ (depending on the gene) and $40 \mathrm{~s}$ at $72^{\circ} \mathrm{C}$. Quantitative PCR progression was monitored by the LineGene9600 (Bioer, Binjiang, China). Dissociation curves served as DNA quality validation as described in (27). GAPDH expression was used as intern control.

\section{Statistical analysis}

The analysis was performed with GraphPad Prism 5. Data were evaluated using non-parametric tests after Shapiro-Wilk test defined that the distribution of data was not normal. Mann-Whitney U test (when two groups of data were compared) and Kruskall-Wallis analysis of variance (when comparing more than two groups) were used to evaluate the significance of mRNA expression and Knosp grade associations. Correlation between angiogenic factors with Knosp grade or the age of patients were determined by Spearman correlation test. In all cases, a $p$ value $<0.05$ was considered statistically significant.

\section{Results}

\section{Endocan is expressed at variable levels in pituitary tumors}

Endocan, an angiogenic marker proposed to be associated with tumor development and progression was analyzed in the pituitary tumors of our cohort. We found that 21 of 28 tumors expressed ESM1, and its mRNA expression levels in the analyzed tumors, were variable. Surprisingly, in some tumor samples no expression of this gene was detected (ND) (Fig. 1).

\section{FGF2 but not VEGF is overexpressed in ESM1-positive pituitary tumors}

As stated above, $25 \%$ of the samples did not express ESM1 (Fig. 1). Therefore, samples were grouped in ESM1-positive and ESM1-negative tumors, and the mRNA levels of the angiogenic biomarkers VEGF, FGF2 and PDGF were evaluated in both groups.

Interestingly, FGF2 mRNA levels were higher in ESM1-positive compared to ESM1-negative tumors (Fig. 2B; $p=0.0415$ ). Conversely, no differences were found between both groups for VEGF (Fig. 2A, NS) or PDGF expression (Fig. 2C, NS).

\section{Correlation between FGF2 and PDGF}

Because an opposite trend of expression was observed in PDGF compared to FGF2 (Figs. 2B and C), we evaluated $F G F 2$ and $P D G F$ correlation in pituitary tumors and found a marked significant negative correlation in the expression of these genes, which may be an indication that both genes are oppositely regulated in pituitary tumors (Fig. 2D; $r=0.63$ and $p=0.0049$ ). ESM1 expression did not correlate with the rest of the angiogenic factors (not shown).

Correlation analysis between ESM1 expression in pituitary tumors and Knosp grade 
We classified tumors according to their cavernous sinus invasion in the five grades defined by Knosp grading system. This classification system divides tumors in $0-4$ grade under the criteria of tumor passing or not the intercarotid line which is a line drawn between the centers of the intra and supra cavernous sinus carotid arteries. It considers grades $0-2$ as non-invasive tumors, and grades 3-4 as invasive tumors (examples in Fig. 3A-B). ESM1 mRNA expression distribution did not show any significant differences in relation to the 0-4 Knosp grade (Fig. 4A). Moreover, no differences in ESM1 mRNA expression were observed when tumor samples were grouped into invasive or not invasive (Fig. 4B). However, a positive correlation between ESM1 and Knosp invasion grade was almost significant (Fig. 4C, $p=0.0526$ ) when the Knosp 4 highest invasive tumors were separated from the cohort. Finally, no differences were observed in ESM1 expression when comparing functioning to non-functioning pituitary tumor samples (Fig. 4D). Moreover, ESM1 expression was not conditioned by the sex of patients or associated with the age at which patients underwent surgery (Supplementary Fig. 1).

\section{Discussion}

Angiogenic markers have been associated with tumor growth and invasiveness in almost every type of cancer and their study provides useful insights of their role in determining tumor behavior. Among these angiogenic and growth factors, the vascular endothelial growth factor (VEGF) is the most important usually studied in oncological malignances, as well as in pituitary tumors (28-30).

It has been demonstrated that VEGF regulates the synthesis and secretion of the endothelial cell-specific molecule-1, endocan or ESM1 (13). Endocan is a secreted proteoglycan considered a biomarker of both neovascularization and tumor progression in hepatocarcinoma and renal carcinoma among other cancers $(11,31)$. Furthermore, not only the pro-angiogenic molecule VEGF but also FGF2 modulate endocan expression (32). Importantly, the dermatan chain of endocan can bind to different growth factors and enhance their biological activity (33), positioning this molecule as an important player in the complex network which regulates angiogenesis and proliferation.

In this context, we evaluated ESM1 mRNA expression in human pituitary tumors and its relation to sinus invasion and angiogenic markers. We determined that not all tumors analyzed expressed this gene, but interestingly, in ESM1-positive samples FGF2 mRNA levels were significantly augmented, suggesting an association of these two angiogenic factors in the tumoral pituitary, as previously described in mesotheliomas (32).

There is growing evidence that under VEGF and FGF2 influence, endocan may promote pituitary tumor cell proliferation as well as endothelial cell growth. Indeed, silencing the endocan gene, Esm 1, in GH3 and MMQ prolactin secreting cells downregulated FGF2 and VEGFR2 among other angiogenic proteins causing reduced cell viability (34). Moreover, the knockdown of Esm1 in HUVEC endothelial cells inhibited tube formation and increased sensitivity to the anti-angiogenic Avastin treatment in vitro, indicating endocan could be a molecular target for pituitary adenoma treatment (34). Additionally, in human pituitary adenoma cells in vitro, silencing FGF2 inhibited cell proliferation and invasion capacity (35). 
Taken together the present and other results point to endocan and FGF2 as potential biomarkers of pituitary tumor progression.

Opposed to the higher FGF2 levels found in ESM1 + samples, PDGF mRNA levels were slightly lower in endocan expressing tumors of our cohort, yielding a strong negative correlation between FGF2 and PDGF expression. There is a scarcity of information on the role of PDGF in the pituitary, and little evidence of PDGF involvement on pituitary adenoma progression. Some studies detected mRNA expression of PDGF and its receptor in human pituitary tissue (36), as well as in the murine folliculo-stellate pituitary cell line TtT/GF cells. In this cell line, PDGF increased VEGF secretion via PDFG receptor (19). Our data show an inverse expression of $F G F 2$ and $P D G F$, which may probably reflect a complex but precise balance of the four angiogenic factors in pituitary tumorigenesis. No previous data in the literature analyzed the relation between endocan and VEGF, FGF2 or PDGF angiogenic factors, and FGF2 and PDGF expression levels were not compared before in pituitary tumor samples. This pattern, showing positive correlation of endocan and FGF2 and inverse correlation of FGF2 and PDGF expression in the pituitary tumor context could be related to the peculiarities that the angiogenic process has in these tumors, as we and other authors already demonstrated $(5,37,38)$. It may be associated to the low grade of malignancy of these tumors in which not all angiogenic factors are simultaneously upregulated.

In turn, endocan association with pituitary tumor invasiveness evidences inconsistencies in the literature. Determined by immunohistochemistry, some authors found that endocan expression in tumor cells but not in endothelial cells, correlated with tumor grade invasion (18). Instead, in another study only endothelial endocan expression correlated with tumor size and recurrence, both signs of aggressiveness, and no correlation with invasion was found (17). Similarly, no relation of endocan and cavernous sinus invasion or resistance to treatment with first-generation somatostatin analogues was found in acromegaly patients (39), though endocan may participate in the resistance of prolactinomas to dopamine therapy (34).

It is worth mentioning, that we evaluated by qRT-PCR the mRNA expression levels of endocan, which identifies the intratumoral ESM1 gene being transcribed. Many reports correlate high ESM1 mRNA levels with poor prognosis and also with metastasis in different cancers such as breast, colon, renal, bladder and others (40-42), but no studies correlating mRNA and aggressiveness in pituitary adenomas have been performed. When we classified tumors by the Knosp grade system ( $0-4$ grade), a correlation between ESM1 and tumor invasion was observed when invasion was considered in the first stages of tumor development, from 0 to 3 Knosp grade in the pituitary tumor cohort $(r=0.464$ and $p=0.0526)$. We do not discard an effect of the small number of invasive tumors in the cohort or heterogeneity within the tumors. Even so, it is probable that ESM1 (probably by its orchestrated relation with VEGF, FGF2 and $P D G F)$ could be implicated in the angiogenic and proliferative process within these tumors in the first period of invasion (eg. passing through 2 to 3 Knosp grade) and endocan would not increase further in the truly invasive phase of pituitary adenomas (Knosp grade 4). Interestingly, Almong et al. described endocan was markedly expressed in concordance with the switch between dormant to fast growing phenotype in experimental angiogenic tumors (15). Moreover, the enhanced FGF2 expression we found in 
ESM1 positive tumors could be supporting the fast-growing state in these tumors as FGF2 has been linked to invasion in cancer and in pituitary adenomas as well $(35,43)$.

Antiangiogenic therapy has been used to treat aggressive pituitary tumors in humans $(22,23)$, and many studies were performed in experimental models, mainly with anti-VEGF therapies $(5,44,45)$. The study of the expression and action of different angiogenic biomarkers such as endocan, FGF2 and PDGF, together with VEGF, will contribute in designing targeted treatment of aggressive and resistant pituitary tumors. Importantly, many compensatory mechanisms in tumors may uncover when VEGF pathway is being blocked (46). Treatment of patients with aggressive tumors is therefore challenging since conventional pharmacological agents often fail. Moreover, invasion into the cavernous sinus is frequently cause of incomplete resection, which in turn results in higher recurrence rate and therefore, determining biomarkers of invasion is paramount.

\section{Conclusions}

We describe a peculiar relationship among the angiogenic markers endocan, VEGF, FGF2 and PDGF in pituitary tumors. Moreover, ESM1 mRNA levels correlated with tumor invasiveness when grade 0-3 tumors were considered; suggesting angiogenesis and tumor progression mediated by endocan (probably together with VEGF, FGF2 and decreased PDGF) may participate in the first steps of pituitary tumor invasiveness. Our data position these molecules as possible future targets in pituitary adenoma treatment.

\section{Abbreviations}

ESM1: Endocan, or endothelial cell specific molecule-1

FGF2: fibroblast growth factor 2

GAPDH Glyceraldehyde 3-phosphate dehydrogenase

HUVEC Human umbilical vein endothelial cells

MRI: magnetic resonance image

ND: not determined

NE: no expression found

PDGF: platelet-derived growth factor

VEGF: Vascular endothelial growth factor

VEGFR2: Vascular endotelial growth factor receptor 2 


\section{Declarations}

\section{Ethics approval and consent to participate}

The project was approved by the respective Institutional Ethical Committees from Xuanwu Hospital, Beijing, China (\#2019-03), and from the neurosurgery division of FLENI Institute, Buenos Aires, Argentina (\#04-2918) and every patient was informed about the procedures of the project and approved and signed a written informed consent for participation in this study. Patient privacy was always preserved.

\section{Consent for publication}

Not applicable

Availability of data and materials: The raw datasets analysed during the current study are available from the corresponding author on reasonable request The datasets supporting the conclusions of this article are included within the article and in Supplementary file 1.

\section{Competing interests}

The authors declare that they have no competing interests

\section{Funding}

This work was supported by grants from Agencia Nacional de Promoción Científica y Tecnológica, Argentina: PICT 901-2013; Consejo Nacional de Investigaciones Científicas y Técnicas and Universidad Nacional del Noroeste de la Provincia de Buenos Aires: PIO CONICET-UNNOBA 2015-2016 $15720150100010 C O$ and SIB UNNOBA \#0241/2017. The three grants were awarded to Dr. Carolina Cristina. The work was also supported with Chinese funding from the Xuanwu Hospital, Medical Capital University of Beijing, China.

\section{Authors' contributions}

All authors read and approved final manuscript

PT obtained samples in surgery, interpreted patients' data, and performed PCRs.

SF performed PCRs, analysis of data, major contributor in writing the manuscript.

GD Discussed data, performed statistics.

AC participated in the analysis of data and statistics

AC obtained samples from surgery interpreted patients' data.

GES obtained samples from surgery, interpreted patients' data. 
SIB Participated in the design of the work, discussed and analysed data.

DBV participated in the analysis of data and writing the manuscript

$A B$ discussed data and rationale

CG Analyzed and interpreted the patients' data

FL Analyzed and interpreted the patients' data

QZ Participated in the design of the work; analyzed and interpreted the patients data

CC major contributor the design of the work, analysis of data, and writing the manuscript

\section{References}

1. Scheithauer BW, Kurtkaya-Yapicier O, Kovacs KT, Young WF Jr, Lloyd RV. Pituitary carcinoma: a clinicopathological review. Neurosurgery. 2005;56:1066-74.

2. Chatzellis E, Alexandraki KI, Androulakis II, Kaltsas G. Aggressive Pituitary Tumors Neuroendocrinology. 2015;101:87-104.

3. Hwang J, Seol HJ, Nam DH, Lee JI, Lee MH, Kong DS. Therapeutic Strategy for Cavernous SinusInvading Non-Functioning Pituitary Adenomas Based on the Modified Knosp Grading System. Brain Tumor Res Treat. 2016;4:63-9.

4. Sánchez-Ortiga R, Sánchez-Tejada L, Moreno-Perez O, Riesgo P, Niveiro M, Picó Alfonso AM. Overexpression of vascular endothelial growth factor in pituitary adenomas is associated with extrasellar growth and recurrence. Pituitary. 2013;16:370-7.

5. Cristina C, Luque GM, Demarchi G, Lopez Vicchi F, Zubeldia-Brenner L, Perez Millan MI, et al. Angiogenesis in pituitary adenomas: human studies and new mutant mouse models. Int $\mathrm{J}$ Endocrinol. 2014. doi:10.1155/2014/608497.

6. Mallea-Gil MS, Cristina C, Perez-Millan MI, Villafane AM, Ballarino C, Stalldecker G, et al. Invasive giant prolactinoma with loss of therapeutic response to cabergoline: expression of angiogenic markers. Endocr Pathol. 2009;20:35-40.

7. Cristina C, Perez-Millan Ms, Luque G, Dulce RI, Sevlever G, Berner, Ss, et al. VEGF and CD31 Association in Pituitary Adenomas. Endocr Pathol. 2010;21:154-60.

8. Niveiro $\mathrm{M}$, Aranda $\mathrm{Fl}$, Peiro G, Alenda C, Pico A. Immunohistochemical analysis of tumor angiogenic factors in human pituitary adenomas. Hum Pathol. 2005;36:1090-5.

9. de la Torre NG, Turner HE, Wass JA. Angiogenesis in prolactinomas: regulation and relationship with tumour behaviour. Pituitary. 2005;8:17-23.

10. Grigoriu BD, Depontieu F, Scherpereel A, Gourcerol D, Devos P, Ouatas T, et al. Endocan Expression and Relationship with Survival in Human Non-Small Cell Lung Cancer. Clin Cancer Res. 2006;12:4575-82. 
11. Leroy X, Aubert S, Zini L, Franquet H, Kervoaze G, Villers A, et al. Vascular endocan (ESM-1) is markedly overexpressed in clear cell renal cell carcinoma. Histopathology. 2010;56:180-7.

12. Chen LY, Liu X, Wang SL, Qin CY. Over-expression of the Endocan gene in endothelial cells from hepatocellular carcinoma is associated with angiogenesis and tumour invasion. J Int Med Res. 2010;38:498-510.

13. Maurage CA, Adam E, Mineo JF, Sarrazin S, Debunne M, Siminski RM, et al. Endocan expression and localization in human glioblastomas. J Neuropathol Exp Neurol. 2009;68:633-41.

14. Atukeren P, Kunbaz A, Turk O, Kemerdere R, Ulu MO, Turkmen Inanir N, et al. Expressions of Endocan in Patients with Meningiomas and Gliomas. Dis Markers. 2016. doi:10.1155/2016/7157039.

15. Almog N, Ma L, Raychowdhury R, Schwager C, Erber R, Short S, et al. Transcriptional switch of dormant tumors to fast-growing angiogenic phenotype. Cancer Res. 2009;69:836-44.

16. Matano F, Yoshida D, Ishii Y, Tahara S, Teramoto A, Morita A. Endocan, a new invasion and angiogenesis marker of pituitary adenomas. J Neurooncol. 2014;117:485-91.

17. Cornelius A, Cortet-Rudelli C, Assaker R, Kerdraon O, Gevaert MH, Prevot V, et al. Endothelial expression of endocan is strongly associated with tumor progression in pituitary adenoma. Brain Pathol. 2012;22:757-64.

18. Miao $Y$, Zong M, Jiang T, Yuan X, Guan S, Wang Y, et al. A comparative analysis of ESM-1 and vascular endothelial cell marker (CD34/CD105) expression on pituitary adenoma invasion. Pituitary. 2016;19:194-201.

19. Kowarik M, Onofri C, Colaco T, Stalla GK, Renner U. Platelet-derived growth factor (PDGF) and PDGF receptor expression and function in folliculostellate pituitary cells. Exp Clin Endocrinol Diabetes. 2010;118:113-20.

20. Board R, Jayson GC. Platelet-derived growth factor receptor (PDGFR): a target for anticancer therapeutics. Drug Resist Updat. 2005;8:75-83.

21. Knosp E, Steiner E, Kitz K, Matula C. Pituitary adenomas with invasion of the cavernous sinus space: a magnetic resonance imaging classification compared with surgical findings. Neurosurgery. 1993;33:610-7.

22. Touma W, Hoostal S, Peterson RA, Wiernik A, SantaCruz KS, Lou E. Successful treatment of pituitary carcinoma with concurrent radiation, temozolomide, and bevacizumab after resection. J Clin Neurosci. 2017;41:75-7.

23. Ortiz LD, Syro LV, Scheithauer BW, Ersen A, Uribe H, Fadul CE, et al. Anti-VEGF therapy in pituitary carcinoma. Pituitary. 2012;15:445-9.

24. Mete O, Lopes MB. Overview of the 2017 WHO Classification of Pituitary Tumors. Endocr Pathol. 2017;28:228-43.

25. Perrone S, Zubeldia-Brenner L, Gazza E, Demarchi G, Baccarini L, Baricalla A, et al. Notch system is differentially expressed and activated in pituitary adenomas of distinct histotype, tumor cell lines and normal pituitaries. Oncotarget. 2017;8:57072-88. 
26. Zubeldia-Brenner L, De Winne C, Perrone S, Rodriguez-Segui SA, Willems C, Ornstein AM, et al. Inhibition of Notch signaling attenuates pituitary adenoma growth in Nude mice. Endocr Relat Cancer. 2019;26:13-29.

27. Garcia-Tornadu I, Diaz-Torga G, Risso GS, Silveyra P, Cataldi N, Ramirez MC, et al. Hypothalamic orexin, $\mathrm{OX} 1$, alphaMSH, NPY and MCRs expression in dopaminergic D2R knockout mice. Neuropeptides. 2009;43:267-74.

28. Costache MI, loana M, lordache S, Ene D, Costache CA, Saftoiu A. VEGF Expression in Pancreatic Cancer and Other Malignancies: A Review of the Literature. Rom J Intern Med. 2015;53:199-208.

29. Weathers SP, de Groot J. VEGF Manipulation in Glioblastoma. Oncology (Williston Park). 2015;29:720-7.

30. Turner HE, Harris AL, Melmed S, Wass JA. Angiogenesis in endocrine tumors. Endocr Rev. 2003;24:600-32.

31. Kang YH, Ji NY, Lee Cl, Lee HG, Kim JW, Yeom YI, et al. ESM-1 silencing decreased cell survival, migration, and invasion and modulated cell cycle progression in hepatocellular carcinoma. Amino Acids. 2011;40:1003-13.

32. Schelch K, Wagner C, Hager S, Pirker C, Siess K, Lang E, et al. FGF2 and EGF induce epithelialmesenchymal transition in malignant pleural mesothelioma cells via a MAPKinase/MMP1 signal. Carcinogenesis. 2018;39:534-45.

33. Sarrazin S, Lyon M, Deakin JA, Guerrini M, Lassalle P, Delehedde M, et al. Characterization and binding activity of the chondroitin/dermatan sulfate chain from Endocan, a soluble endothelial proteoglycan. Glycobiology. 2010;20:1380-8.

34. Cai L, Leng ZG, Guo YH, Lin SJ, Wu ZR, Su ZP, et al. Dopamine agonist resistance-related endocan promotes angiogenesis and cells viability of prolactinomas. Endocrine. 2016;52:641-51.

35. Zhou K, Fan YD, Duysenbi S, Wu PF, Feng ZH, Qian Z, et al. siRNA-mediated silencing of bFGF gene inhibits the proliferation, migration, and invasion of human pituitary adenoma cells. Tumour Biol. 2017. doi:10.1177/1010428317704805.

36. Leon SP, Carroll RS, Dashner K, Glowacka D, Black PM. Messenger ribonucleic acid expression of platelet-derived growth factor subunits and receptors in pituitary adenomas. J Clin Endocrinol Metab. 1994;79:51-5.

37. Ms P-M, Ss B, Luque G, Bonis C, Sevlever G, Becu-Villalobos D, et al. Enhanced nestin expression and small blood vessels in human pituitary adenomas. Pituitary. 2013;16:303-10.

38. Itoh J, Serizawa A, Kawai K, Ishii Y, Teramoto A, Osamura RY. Vascular networks and endothelial cells in the rat experimental pituitary glands and in the human pituitary adenomas. Microsc Res Tech. 2003;60:231-5.

39. Iacovazzo D, Chiloiro S, Carlsen E, Bianchi A, Giampietro A, Tartaglione T, et al. Tumour-infiltrating cytotoxic T lymphocytes in somatotroph pituitary neuroendocrine tumours. Endocrine. 2020;67:6518. 
40. Rennel E, Mellberg S, Dimberg A, Petersson L, Botling J, Ameur A, et al. Endocan is a VEGF-A and $\mathrm{PI} K \mathrm{~K}$ regulated gene with increased expression in human renal cancer. Exp Cell Res. 2007;313:128594.

41. Roudnicky F, Poyet C, Wild P, Krampitz S, Negrini F, Huggenberger R, et al. Endocan is upregulated on tumor vessels in invasive bladder cancer where it mediates VEGF-A-induced angiogenesis. Cancer Res. 2013;73:1097-106.

42. Zuo L, Zhang SM, Hu RL, Zhu HQ, Zhou Q, Gui SY, et al. Correlation between expression and differentiation of endocan in colorectal cancer. World J Gastroenterol. 2008;14:4562-8.

43. Santolla MF, Vivacqua A, Lappano R, Rigiracciolo DC, Cirillo F, Galli GR, et al. GPER Mediates a Feedforward FGF2/FGFR1 Paracrine Activation Coupling CAFs to Cancer Cells toward Breast Tumor Progression. Cells. 2019;8.

44. Luque GM, Perez-Millan MI, Ornstein AM, Cristina C, Becu-Villalobos D. Inhibitory Effects of Antivascular Endothelial Growth Factor Strategies in Experimental Dopamine-Resistant Prolactinomas. J Pharmacol Exp Ther. 2011;337:766-74.

45. Korsisaari N, Ross J, Wu X, Kowanetz M, Pal N, Hall L, et al. Blocking vascular endothelial growth factor-A inhibits the growth of pituitary adenomas and lowers serum prolactin level in a mouse model of multiple endocrine neoplasia type 1. Clin Cancer Res. 2008;14:249-58.

46. Zhao Y, Adjei AA. Targeting Angiogenesis in Cancer Therapy: Moving Beyond Vascular Endothelial Growth Factor. Oncologist. 2015;20:660-73.

\section{Figures}




\section{ESM1}

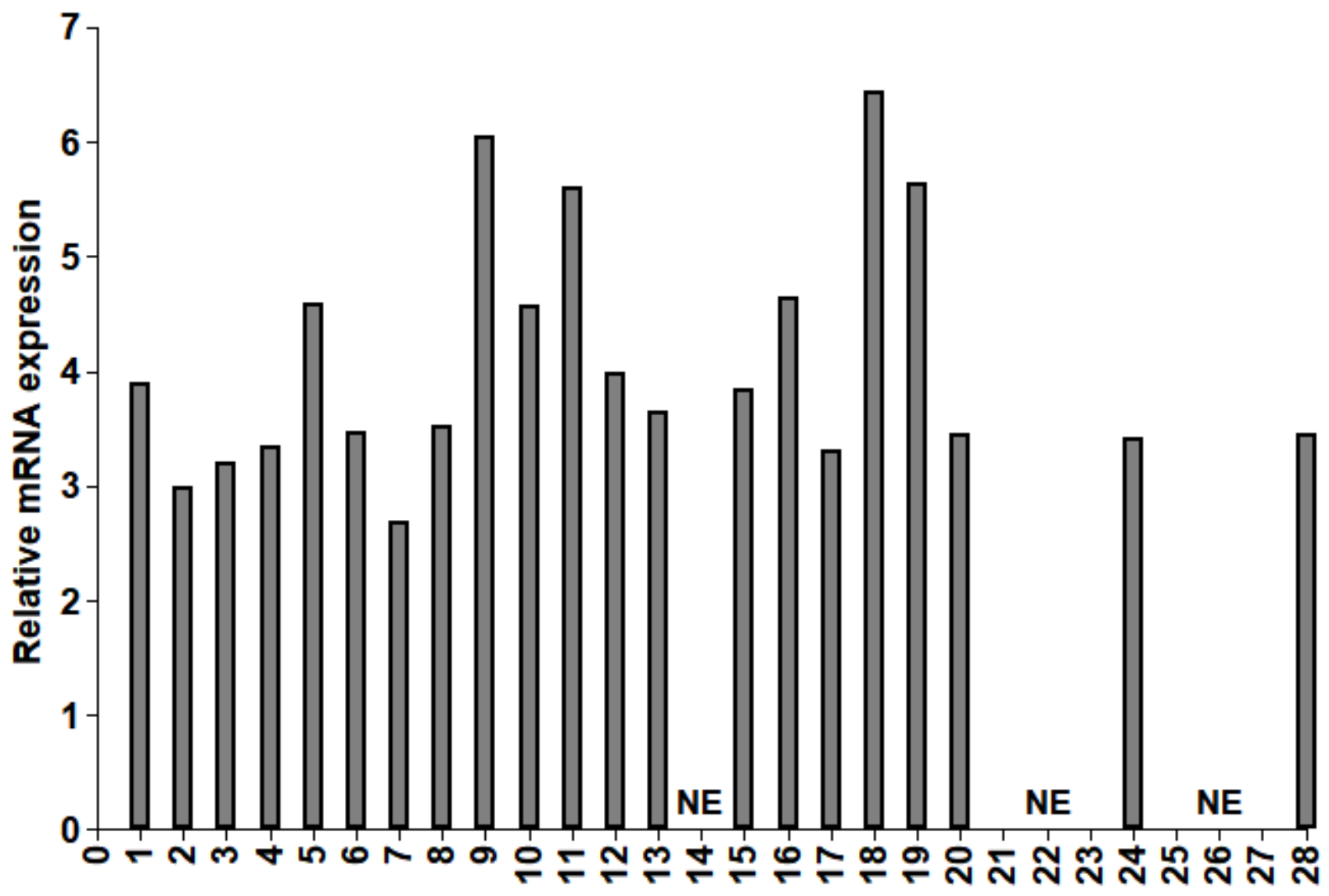

Figure 1

Endocan expression in pituitary tumor samples. mRNA expression of ESM1 gene was determined by qRTPCR. Gene levels are shown normalized to the housekeeping gene GADPH levels. $(N=28, N E=N o$ expression found). 
A

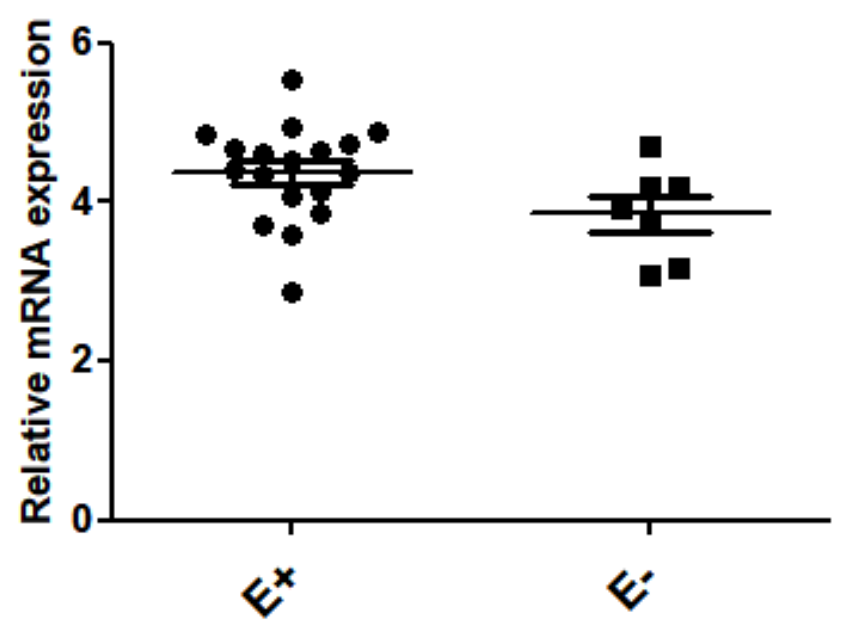

C

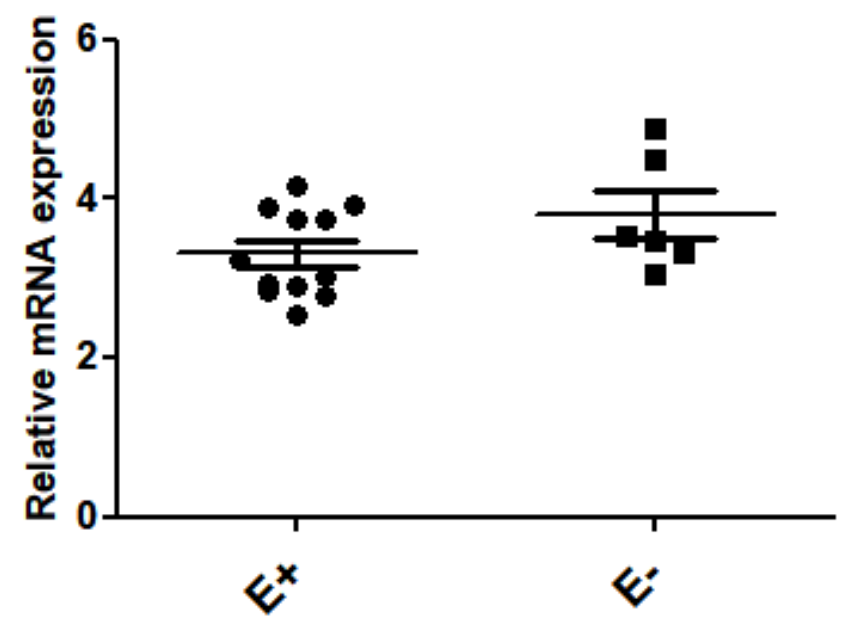

B

FGF2

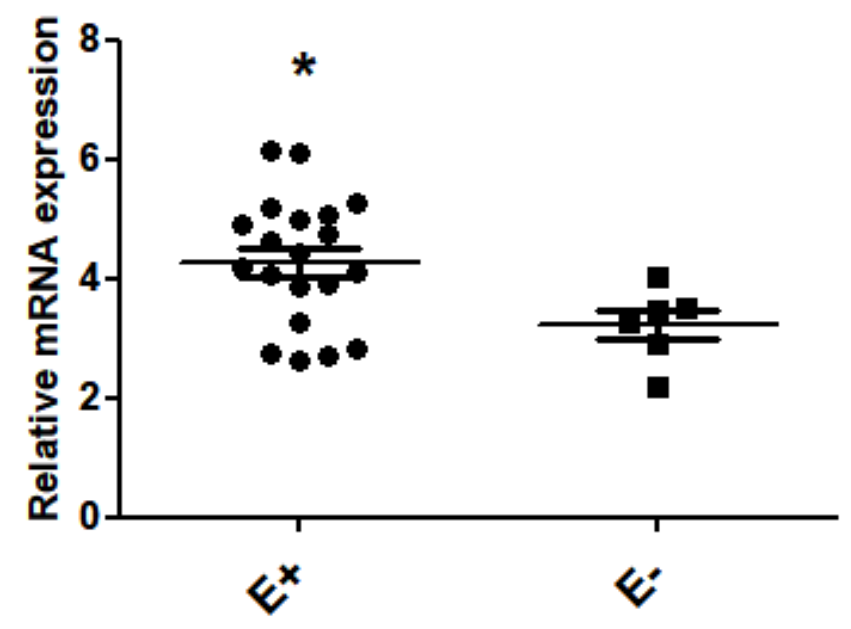

D PDGF-FGF2 correlation

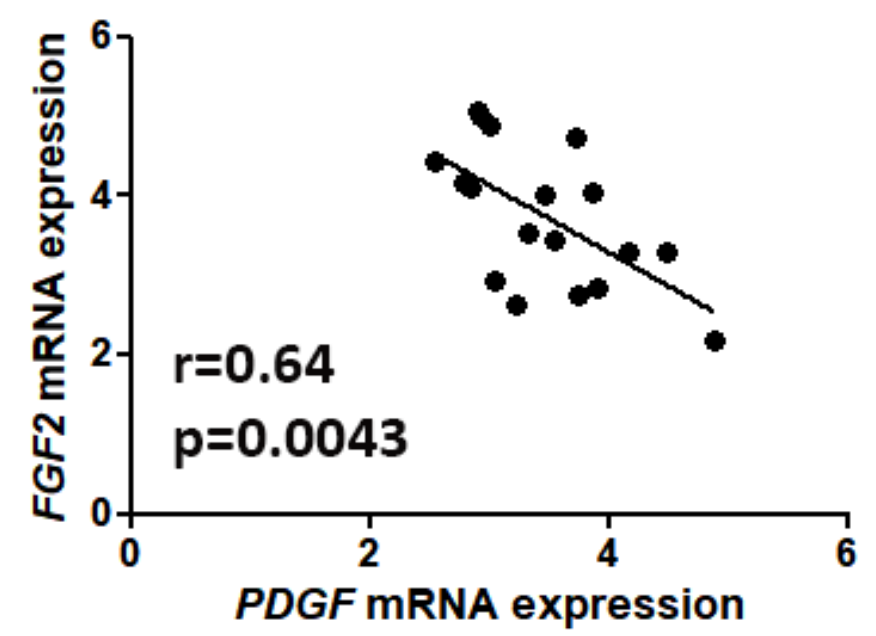

Figure 2

ESM1-positive pituitary tumors have higher expression levels of FGF2. mRNA expression of VEGF (A), FGF2 (B) and PDGF (C) genes determined by qRT-PCR in ESM1-positive (E+) and ESM1-negative (E-) pituitary tumors. Gene levels are normalized to the housekeeping gene GADPH. A) $N=18,7$ and $p=0.084$; B) $N=20 ; 6$ and $p=0.041$. C) $N=12 ; 6$ and $p=0.174$. D) Relation between mRNA levels of FGF2 and PDGF was determined, $\mathrm{N}=18$. FGF2 and PDGF correlate negatively. The Spearman coefficient of correlation and $p$ value are shown in the graph $(r=0.63, p=0.0049)$. 

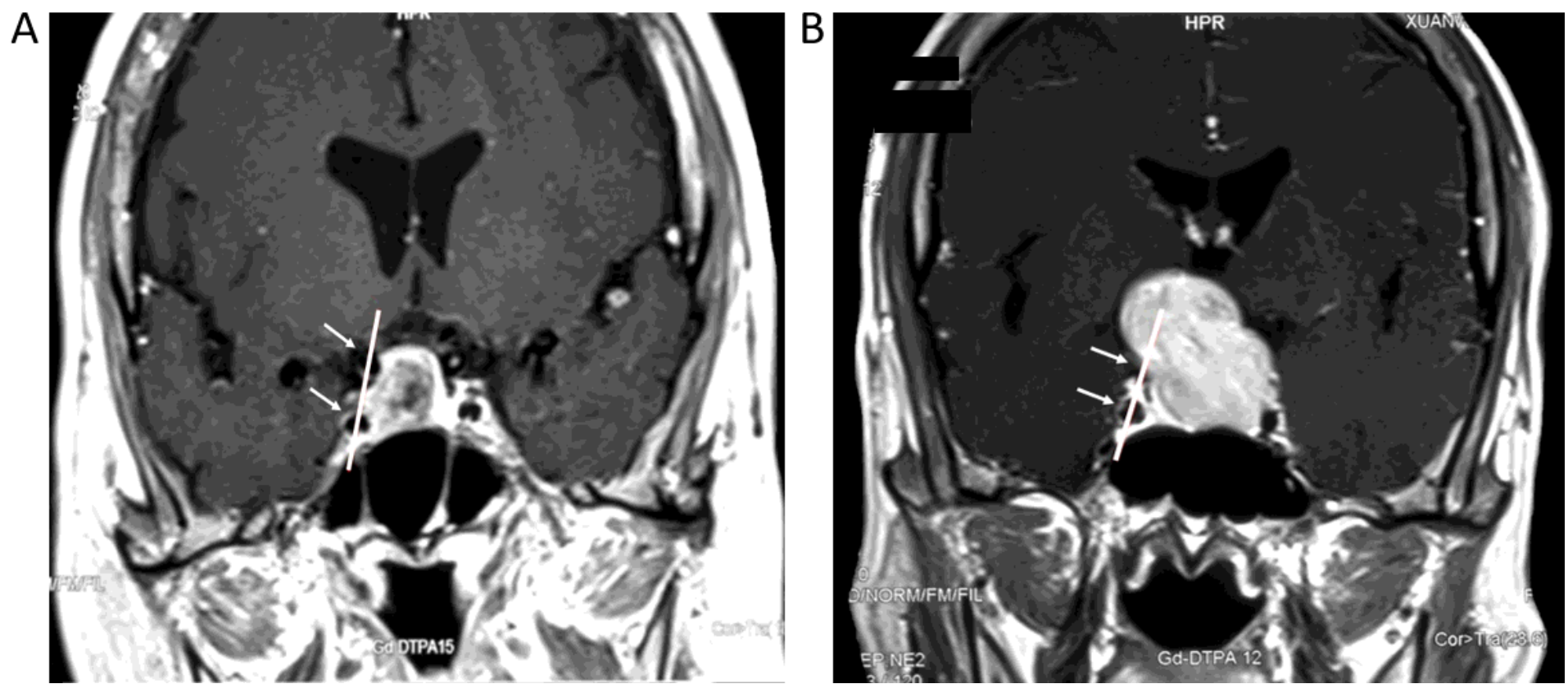

\section{Figure 3}

Representative images of patient's MRI for Knosp grade determination. A) Coronal section MRI of a Knosp grade 1 (noninvasive) macroadenoma, and B) a Knosp grade 4 (invasive) macroadenoma. Knosp grade classification of the cavernous sinus invasion based on an imaginary line (white) that passes through both internal carotid arteries. White arrows show internal carotid arteries. 
A

ESM1

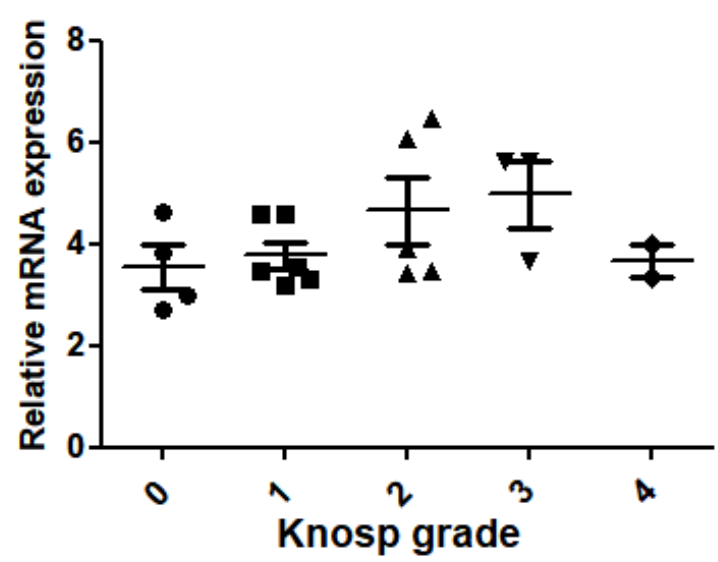

C

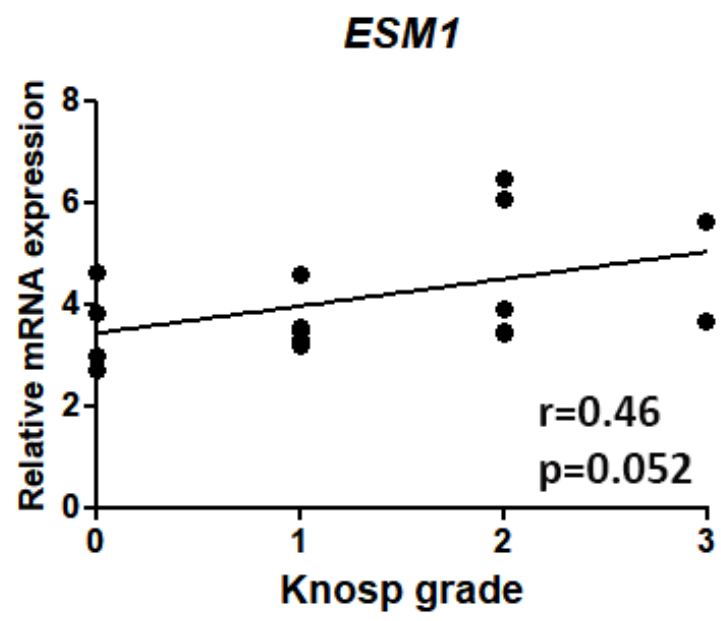

B

ESM1

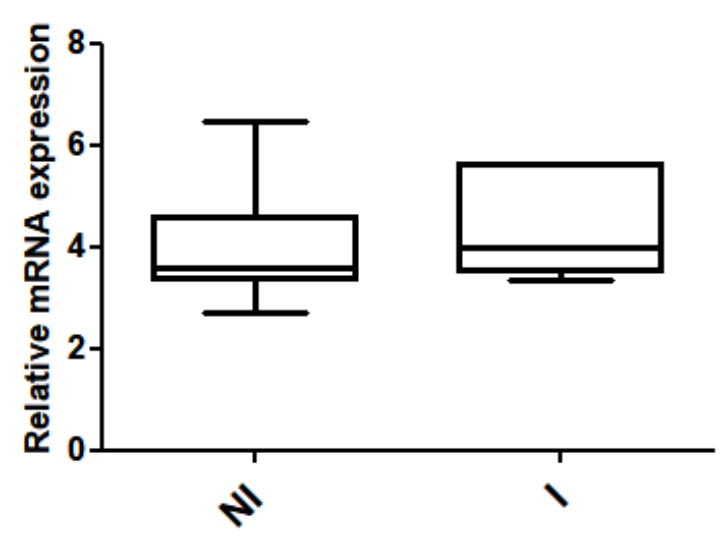

D

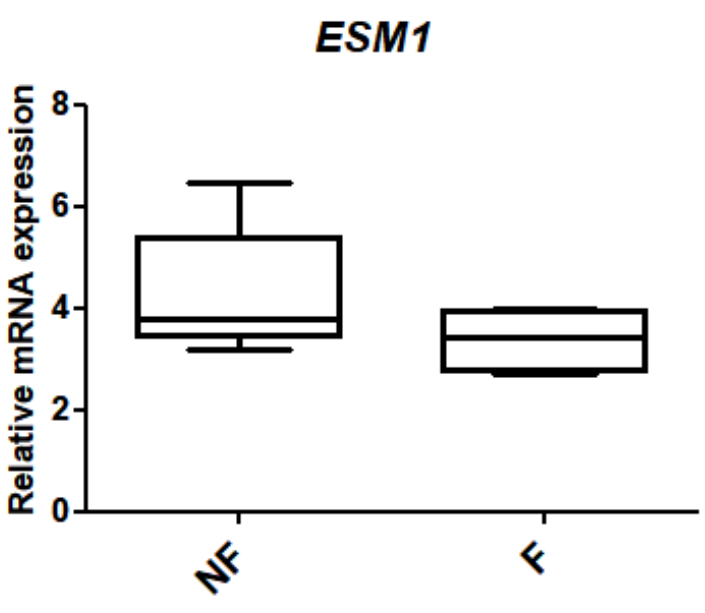

- Median

25\%-75\%

工 Min-Max

Figure 4

Endocan expression according to Knosp grade, invasiveness and histotype of pituitary tumors. A) Relation between ESM1 mRNA expression and Knosp grade classification (0-4) determined by magnetic resonance imaging (MRI) ( $=20 ; p>0.05)$. B) Box graphic of ESM1 mRNA expression classified by tumor invasiveness ( $\mathrm{Nl}=$ Non Invasive; $\mathrm{I}=$ Invasive. $\mathrm{N}=16 ; 5$ respectively. $\mathrm{p}>0.05)$. C) Correlation between ESM1 and Knosp grade considering 0-3 grades $(N=18 ; r=0.464 ; p=0.0526)$. D) Box graphic of ESM1 mRNA expression classified by tumor histotype ( $N F=$ Non Functioning; $F=$ Functioning. $N=15 ; 4$ respectively. $\mathrm{p}>0.05)$.

\section{Supplementary Files}

This is a list of supplementary files associated with this preprint. Click to download.

- SupplementaryFig.12.tif 